\title{
On the Characteristics of Circumstellar Envelopes in Be Stars near the Central Object
}

\author{
A. Moujtahid \\ Observatoire de Paris-Meudon, DASGAL/UMR 8633 du CNRS, \\ F-92195 Meudon Principal Cedex, France
}

\section{J. Zorec}

Institut d'Astrophysique de Paris, CNRS, 98 bis bd. Arago, F-75014 Paris, France

\author{
A.M. Hubert \\ Observatoire de Paris-Meudon, DASGAL/UMR 8633 du CNRS, \\ F-92195 Meudon Principal Cedex, France
}

\begin{abstract}
Observed visible energy distributions of Be stars were studied with analytic flux distributions produced by flattened circumstellar envelopes. The theoretical representation of emitted fluxes also allows for density distribution effects in the region where the visible continuum emission/absorption is raised. Two main conclusions were reached: (a) regions responsible for spectrophotometric "shell" phases have, on average, steeper density distributions than those producing the spectrophotometric "Be" phases; (b) the mean temperatures of the studied circumstellar envelope regions shows a linear relation with their mean "inverse" extent $R_{*} / R$.
\end{abstract}

\section{Introduction}

Although numerous authors have already discussed temperatures, extents and opacities of regions in the circumstellar envelope (CE) responsible for the visible $(\lambda \lambda 3000-8000 \AA)$ energy distributions in Be stars, fewer works deal with density distribution in such regions. Knowing that for a given opacity and temperature, the amount of energy emitted compared to that absorbed depends on the extent of the radiating zone, some characteristics of the observed energy distribution may be related to the density distribution in the CE. Two spectrophotometric phases or types of energy distribution are distinguished in Be stars, depending on whether continuum emission or absorption dominates. In the first case, we shall refer to the SPh-E (spectrophotometric emission phase) when the second component of the Balmer discontinuity (BD) is in emission. In the second case, the SPh-A (spectrophotometric absorption phase), the second component of the $\mathrm{BD}$ is in absorption. Assuming that the density distribution in the zones where the continuum spectrum is formed follows a power law $\rho \sim R^{-\gamma}$, it is important to derive the exponent $\gamma$ from observations, to find out whether different spec- 
trophotometric phases are related or not to different density distribution in the CE. In fact, it is expected that the extent of the region in the CE producing the continuum spectrum is related to the value of $\gamma$.

\section{The model of the CE}

Observations (Stee et al. 1998) have shown that the extent of the region producing the visible energy distribution in $\gamma$ Cas is $R \sim 1.6 R_{*}$, half of the extent predicted by disc-like models of $\mathrm{CE}$. The electron density in these regions cannot be higher than $N_{\mathrm{e}} \sim 10^{13} \mathrm{~cm}^{-3}$ because otherwise the second BD would overlap the photospheric $\mathrm{BD}$ at much longer wavelengths than actually observed. On the other hand, to gain enough emission or absorption in the continuum spectrum, the region that forms them must cover a significant fraction of the stellar surface. In such a picture there is no reason for the regions in CE near the central star to be strongly flattened. In such a case an equivalent spherical-like formulation of the $\mathrm{CE}$, allowing however for some flattening and for the related aspect angle effects, may be suitable to describe the observed energy distributions. In Moujtahid et al. (1999) it was shown that a slab-like formulation of the CE near the central star, which allows for flattening and aspect angle effects in the opacities and in the emitting/absorption effective surface of the studied region, produces energy distributions closely resembling those obtained from more detailed Monte Carlo simulations in ellipsoidal CE. An observed energy distribution can then be described with 5 independent parameters: $R=R_{\mathrm{e}} \Lambda^{1 / 2}$, the reduced extent of the zone in the $\mathrm{CE}(E \leq \Lambda \leq 1=$ flattening factor which is a function of the aspect angle $i$ and ellipticity $E ; R_{\mathrm{e}}=$ actual mean extent of the CE); $T_{\text {env }}$, the mean temperature of the region; its scattering opacity $\overline{\tau_{\mathrm{e}}}=\tau_{\mathrm{e}} / \Lambda$ and the absorption (bf $+\mathrm{ff}$ ) opacity $\overline{\tau^{\mathrm{V}}}=\tau^{\mathrm{V}} / \Lambda$ at $\lambda=0.56 \mu \mathrm{m}$ and the exponent $\gamma$ of the density distribution law in the studied $\mathrm{CE}$ zone.

The energy emitted by the star-CE system is given by an analytic expression of the type:

$$
F_{\lambda}=\left(\frac{R_{*}}{D}\right)^{2} \alpha_{\lambda}\left(R, \tau_{\lambda}\right) F_{\lambda}^{*}+\left(\frac{R}{D}\right)^{2} \beta_{\lambda}\left(R, \tau_{\lambda}\right) S_{\lambda}\left(R, T_{\text {env }}, \tau_{\lambda}\right)
$$

where $R_{*}$ is the stellar radius, $D$ is the distance of the star, $F_{\lambda}^{*}$ is the stellar flux, $R$ is the reduced mean extent of the region analysed, $\tau_{\lambda}$ is the total continuum opacity of the region and $S_{\lambda}$ is its source function for continuum radiation: $S_{\lambda}=\left(1-\epsilon_{\lambda}\right) B_{\lambda}\left(T_{\text {env }}\right)+\epsilon_{\lambda} J_{\lambda} ; \epsilon_{\lambda}$ is the ratio of scattering to the absorption coefficient, $B_{\lambda}$ is the Planck function and $J_{\lambda}$ is the mean intensity of radiation. $\alpha_{\lambda}$ and $\beta_{\lambda}$ are functions of the opacity $\tau_{\lambda}$ through exponential integrals or order $3 E_{3}$ and of $\mu_{o}=\left[1-\left(R_{*} / R\right)^{2}\right]^{1 / 2}$. In the limits for $R_{*} / R \rightarrow 0$ and $R_{*} / R \rightarrow 1$, expression (1) recovers the forms most currently used, respectively:

$$
\begin{aligned}
& F_{\lambda} \times(D / R)^{2}=F_{\lambda} e^{-\tau_{\lambda}}+\left(R / R_{*}\right)^{2} S_{\lambda}\left[1-2 E_{3}\left(2 \tau_{\lambda}\right)\right] \\
& F_{\lambda} \times(D / R)^{2}=2 E_{3}\left(\tau_{\lambda}\right) F_{\lambda}+\left(R / R_{*}\right)^{2} S_{\lambda}\left[1-2 E_{3}\left(\tau_{\lambda}\right)\right]
\end{aligned}
$$




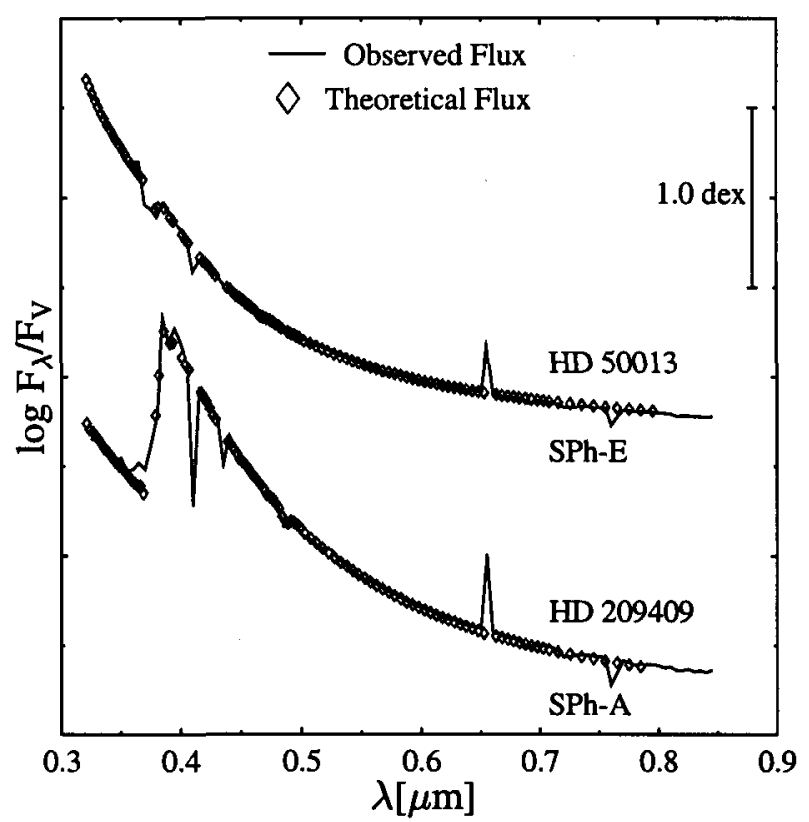

Figure 1. Fit of energy distributions observed in two Be stars with theoretical flux distributions given by relation (1). HD 50013 is in a $\mathrm{SPh}-\mathrm{E}$ phase and HD 209409 is in a SPh-A phase

\section{Observational data}

A sample of 21 Be stars, whose energy distributions were investigated by Kaiser (1987) and Dachs et al. (1989), was studied in this work.

\section{Results and discussion}

To fit the observed energy distributions we used the "simulated annealing" method (Metropolis et al. 1953), a robust iterative method based on probabilistic predictions of solutions in the space of variables. Some fits obtained in this way are shown in Fig. 1. The mean $\gamma$ exponent of the density distribution obtained for Be stars with the second BD in emission is smaller than for stars where the second $\mathrm{BD}$ is in absorption or close to normal. Stars with the strongest emissions, like HD 28497, HD 63462, HD 66194, HD 110432, HD 148184, etc have the lowest $\gamma$ exponents. On average it is:

$$
<\gamma>_{\mathrm{SPh}-\mathrm{A}} \sim 2<\gamma>_{\mathrm{SPh}-\mathrm{E}}-1
$$

This suggests that the CE regions that produce the SPh-E phases have a less steep density distribution near the central star than those favoring the SPh-A phases. The tendency would then be that $\mathrm{CE}$ with lower $\gamma$ are more extended on average. A difference in the value of $\gamma$ also indicates a difference in the mean 


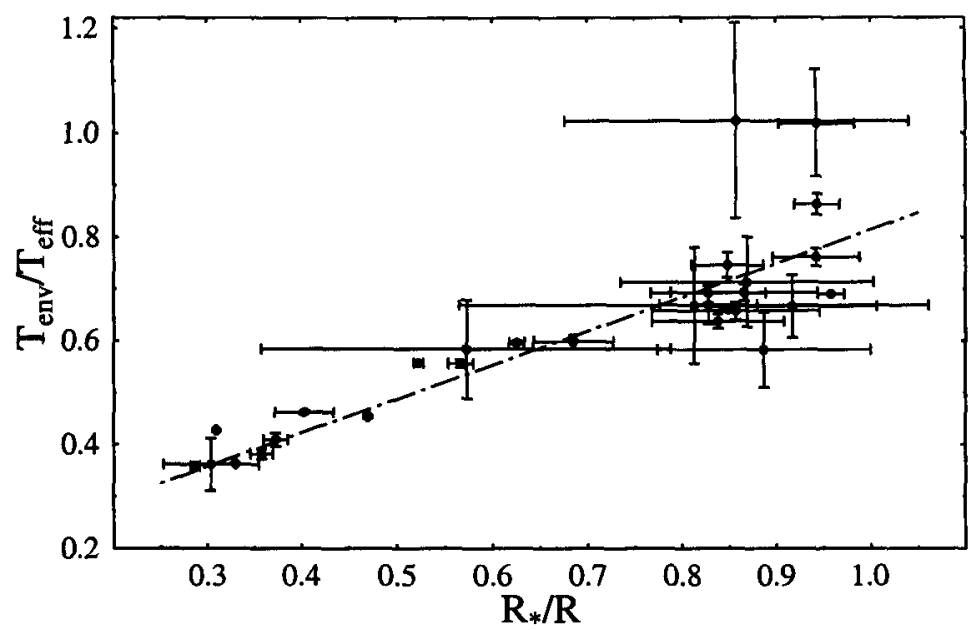

Figure 2. Linear relation between the derived mean temperature $T_{\text {env }} / T_{\text {eff }}$ and the mean "inverse" extent $R_{*} / R$ of the $\mathrm{CE}$ region which produces the visible continuum energy distributions in the studied $\mathrm{Be}$ stars

velocity field near the stars. If the results above are confirmed, $\mathrm{SPh}-\mathrm{E}$ phases would have slightly more outward accelerated movements of material around the star than SPh-A phases.

Another interesting result arising from this study is the relation of $T_{\text {env }} / T_{\text {eff }}$ with the mean "inverse" extent $R_{*} / R$. This relation is shown in Fig. 2. The mean slope reproduces the temperature $T_{\text {env }}$ derived from $B\left(T_{\text {env }}\right)=W\left(R_{*} / R\right) \times$ $B\left(T_{\text {eff }}\right)$ in the interval $0.1 \leq R_{*} / R \leq 1$. The B's are the integrated Planck functions and $\mathrm{W}$ is the geometrical dilution factor. In two stars, where also errors in the $T_{\text {env }}$ are not negligible, the mean CE temperature is of the order of the effective temperature, as if they correspond to regions where the "activity" is high.

\section{References}

Dachs, J., Poetzel, R., Kaiser, D. 1989, A\&AS 78, 487

Kaiser, D. 1987, A\&AS 67, 203

Metropolis, N., Rosenblunth, A., Rosenblunth, M. et al. 1953, J. Chem. Phys. 21,1087

Moujtahid, A., Zorec, J., Hubert, A.M. 1999, A\&A 349, 151

Stee, Ph., Vakili, F., Bonneau, D. et al. 1998, A\&A 332, 268 